\section{Do we really need a new operation for Hirsch- sprung's disease?}

Dear Sir,

I read with interest the original article recently published by Gopal [1], in which the author presented a new surgical technique for the treatment of Hirschsprung's disease. I have some concerns regarding this technique: (1) a fairly long aganglionic segment (up to the level of the pelvic floor) was left in place, aiming to preserve a sensation of defecation. The ectorectal dissection around this segment down to the anorectal junction may affect the nerve supply, which is already abnormal. Also, potential impairment of its blood supply may lead to ischemia, resulting in later fibrosis and stricture; (2) there are considerable technical difficulties in tucking the seromuscular cuff around the rectum low enough to avoid leaving an aganglionic segment without ectorectal seromuscular cuff coverage through a totally pelvic approach; (3) the author's technique still carries the risk of injury to the pelvic nerves during ectorectal dissection; and (4) the author performs a routine back-up colostomy, which could safely be confined only to cases in which intraoperative circumstances necessitate one.

Finally, the potential disadvantages of the author's technique and the fact that the already available classic surgical procedures for the treatment of Hirschsprung's disease have proven to have fairly good short-term as well as comparable long-term results raises the question: do we really need a new operation for Hischsprung's disease?

\section{Essam A. Elhalaby}

C. S. Mott Children's Hospital, L 2110; 0245, University of Michigan Medical Center, Ann Arbor, MI 48109, USA

\section{References}

1. Gopal G (1992) Ectorectal seromuscular colon cuffing. Pediatr Surg Int 7: 444-445

\section{Treatment of choledochal cyst in Kuala Lumpur}

\section{Dear Sir,}

Goon et al., in their article on the treatment of choledochal cyst in Kuala Lumpur [1], reported 4 deaths in 35 patients, 1 of which was attributed to cholangitis, sepsis, and liver failure (the cyst was simply drained into the intestine) and another from postoperative bleeding from the cyst bed (the patient had undergone complete excision of the choledochal cyst and Rouxen-Y choledochojejunostomy).

For some time now we have advocated cyst excision by an internal approach to avoid these types of complications, especially if the cyst appears inflamed or is especially large [2]. In this approach, a plane of dissection is developed between the "mucosal" layer and the fibrous cyst wall. The mucosal layer is excised, leaving the fibrous wall intact. The latter provides a protective layer between the field of

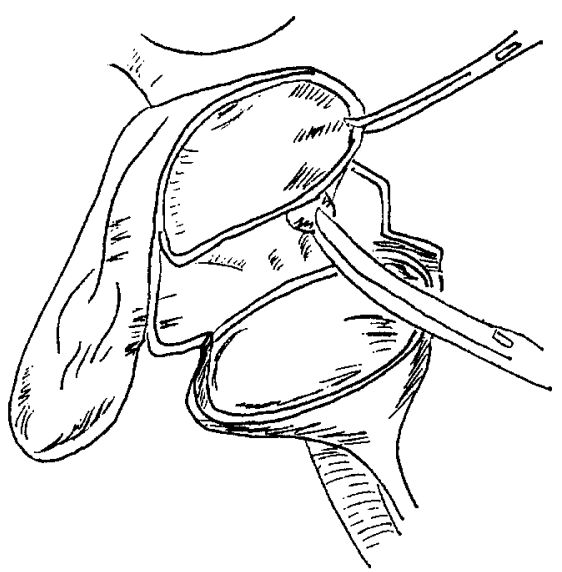

Fig. 1. Internal approach to resection of choledochal cyst. Inner cyst lining is separated from fibrous outer wall by blunt dissection; lining is also stripped distally and distal common bile duct oversewn. Proximally, the plane of dissection is continued until the normal hepatic duct is encountered, at which point the cyst is resected

dissection and the portal structures (Fig. 1), thus avoiding inadvertent major hemorrhage. A direct anastomosis of the hepatic duct to the jejunum (Roux-en-Y) completes the operation. Using this technique, we feel that almost all choledochal cysts can be safely excised.

R. M. Holland and J. R. Lilly

Department of Pediatric Surgery, The Children's Hospital, 1950 Ogden St, B323, Denver, CO 80218, USA

\section{References}

1. Goon HK, Tai A, Samad SA (1992) Congenital bile duct dilatation: review of 35 cases. Pediatr Surg Int 7: 332-336

2. Lilly JR (1979) The surgical treatment of choledochal cyst. Surg Gynecol Obstet 149: 36 